\title{
A COMPARATIVE STUDY OF FRESH AUTOGENOUS AND PRESERVED HOMOGENOUS TENDON GRAFTS IN RABBITS
}

\author{
Lee J. Cordrey, Tampa, Florida \\ Hugh McCorkle and Emmett Hilton, Cleveland, Ohio, United States of America
}

From the Surgical Research Laboratory, Highland View Hospital, Cleveland

The problems of repair and grafting of tendons have received considerable attention. However, conclusions have been hampered by lack of a standard terminology and by attempts to assign the origin of tendon, paratenon, and cicatrix to distinct types of cell. The potentialities of cells under stress have been insufficiently recognised. Beltzow (1883), Viering (1891), Busse (1892), Rehn (1919) and Schwarz (1922) concluded that though tendon stumps took some part in the new formation of tendon, the intervening tissue, despite histological resemblances, was essentially scar and not regenerated tendon. They agreed that the clot at the tendon ends was gradually replaced by granulation tissue from the paratenon. Adams (1860) attributed the regeneration of new tendon to activity by the sheaths rather than by the stumps of the tendons. There has been disagreement about the role of the tendon cells. Busse (1892) described tendon cell proliferation, as did Viering (1891), Enderlen (1893), Seggel (1903), Borst (1903), Imayoshi (1925) and Migliavacca (1925). Mason and Allen (1941) and Peer (1955) thought that the parenchymal cells of autogenous tendon grafts could survive and participate in the process of repair. However, Bizzozero (1868), Schradieck (1900), Marchand (1901), Schwarz (1922), Bloch and Bonnet (1929) and Lange (1929) concluded that tendon cell proliferation played little or no part in healing tendon defects. Skoog and Persson (1954) maintained that degenerative changes were inevitable in autogenous tendon transplants, and that replacement by invading cells from the host tissues must occur before healing was complete. Viering (1891) was the first to note that, with use, nuclei and fibres of tendon lined up in rows parallel to the line of pull. Kirschner (1909) studied autogenous grafts of tendon and fascia and found that tendon, whether functioning or not, remained at least partly alive but that its fibres tended to lose their normal parallel arrangement. Gallie and LeMesurier (1922) stated that after tendon injury healing ultimately occurred by the production of ordinary scar, tendon cells and fibres apparently taking no active part in repair. There was no proliferation of the tendon cells themselves. Mayer (1921) stated that in the free transfer of a tendon the blood supply was destroyed and the vitality of the tendon was entirely dependent on the accuracy of coaptation. Neuhof (1923) thought that all the transplanted tendon tissue died and was replaced by cellular proliferation from surrounding tissues. Mason and Shearon (1932) found, with autogenous tendon grafts, that during the first two weeks grafts and stumps were united by proliferation of paratenon or peritendinous tissues; this phase overlapped that of tenoblastic proliferation which began at the fourth or fifth day after grafting. Twelve to fourteen days after grafting, tenoblasts proliferating in the graft extended into the stumps if apposition had been good. This nuclear increase in the graft was present in longitudinal strips or oval zones. After the second week healing was produced by organisation of the scar between the ends of graft and stumps, in the formation of which the tenoblasts themselves played the most important part. During the fourth and fifth weeks after operation the sheath separated progressively more easily from the organising tendon and a well organised tendon with a paratenon arrangement about it was eventually produced. They could not demonstrate any new synovial sheath formation. Teneff and Fonda (1953) found no differences in the rate, completeness, or character of growth in autogenous, homogenous, or heterogenous grafts of frozen tendon. They thought that the fibrillar portion 
of the graft acted as a scaffolding for newly formed embryonal fibroblasts. Vacirca (1954) noted that heterogenous grafts kept at 38 degrees Fahrenheit (3.3 degrees Centigrade) for up to ninety days " took" after suture followed by immobilisation for fifteen to twenty days. Graham (1955) found that homografts of tendon kept at 10 degrees Fahrenheit for three months appeared like normal tendon forty-seven days after transplantation. He thought that the homografts were probably replaced by living tendon. Peacock (1959) thought that heterologous and homologous tendon grafts were primarily collagen transplants. He thought that they represented purely homostatic grafts which were incorporated into the host only after destruction and replacement by host cells of all transferred cells. He supposed that the transplanted fibroblasts were killed by cytotoxic antibodies. Later, however (Peacock and Petty 1960), he could not demonstrate clinically significant antigens in whole tendons or any of their components. Flynn, Wilson, Child and Graham (1960) recently compared heterogenous tendon grafts preserved in homologous plasma, and irradiated, frozen heterogenous tendon grafts, with autogenous tendon transplants. They stated that preserved heterogenous tendons caused reactions differing only slightly from those caused by autogenous tendon transplants. All their transplants underwent necrosis, and they concluded that the necrotic transplants acted as struts and were replaced by tenoblasts from the host stumps.

Though it appears that refrigerated tendon grafts at least are incorporated into a host in a manner similar to fresh grafts it remains uncertain whether such grafts can be used in clinical practice. Bunnell (1956) stated that a preserved graft was yellowish in colour, that it stained like fixed tissue and that it provoked more foreign body reaction and became more adherent than did a fresh graft. It was therefore much less satisfactory than a live autogenous graft.

The present study was undertaken to determine possible variation in vascularisation, degree of peripheral reaction and relative healing time of unpreserved autogenous tendon grafts and of homogenous tendon grafts, preserved otherwise than by refrigeration.

\section{MATERIAL AND METHODS}

The anatomical arrangement of the tendo calcaneus of the rabbit closely resembles that of true synovial tendons (Nisbet 1960). There is a strong fascial sheath about the combined tendons which constitute the tendo calcaneus. The tendon itself is covered by a thin, glistening epitenon and the space between sheath and tendon contains fat only in relation to the mesotenon. In the experiments to be described the sheath was not closed, so the result of healing should resemble those seen after placing a flexor tendon graft in the hand.

Sixty-eight rabbits were used. 000 black silk sutures were used, placed in figure of eight fashion in the tendon ends. Extra silk sutures were placed at the periphery of the joins. After operation the limb was immobilised in a plaster extending to the groin. An attempt was made to keep the tendon relaxed while the plaster was applied. The plasters were kept on until the animal was killed so as to keep uniform control of the use of the limb.

Autogenous grafts - In twenty-six animals a segment of soleus tendon about four centimetres long was excised, turned round end to end and resutured. No attempt was made to preserve the sheath. Animals were killed daily from the first to the fourteenth day after operation. Thereafter they were killed at three- or four-day intervals to the end of the sixth week after operation. Soon after death the limb was incised in the line of the scar and the tendon was examined and photographed. The whole tendon was then removed and put in formalin. Five animals were eliminated from the series because of infection or obviously inadequate healing of the graft.

Homogenous grafts-In twenty-one rabbits, weighing 3,000 grammes or more, a four-centimetre segment of the right soleus tendon was excised. These segments were prepared as homogenous grafts: seven were preserved in 1:1,000 aqueous merthiolate solution; seven were preserved in 70 per cent ethanol, and seven were lyophilised by the method of Creech, DeBakey, Cooley and Self (1954). In each of twenty-one similar rabbits a four-centimetre segment of the tendo calcaneus was removed and a graft of one of the three types was sutured into the gap so produced. One animal from each of the three groups was killed each week for seven weeks and the soleus tendon and its graft resected.

VOL. 45 B, NO. 1, fEBRUARY 1963 


\section{RESULTS}

AUTOGENOUS, NON-PRESERVED TENDON GRAFTS

Two days-The host tendon stumps were rounded, the surfaces of the graft were covered with a network of fine capillaries, and the graft appeared slightly oedematous and thickened. There was no specific vascular pattern over graft or stumps. The space between stumps and graft was filled with clot.

Microscopically there was acute inflammation with haemorrhage and exudation of fibrin and polymorphonuclear leucocytes about the periphery of the graft and between graft and host. There was minimal cellular exudation and slight capillary proliferation in the proximal and distal stumps of the host. Capillary proliferation extended from the host to the surfaces of the graft but not within the graft. The nuclei of the graft cells had granular chromatin and were not pyknotic. They resembled the nuclei within the stumps. The fibres within the graft appeared slightly more eosinophilic than those in the tendon stumps (Fig. 1).

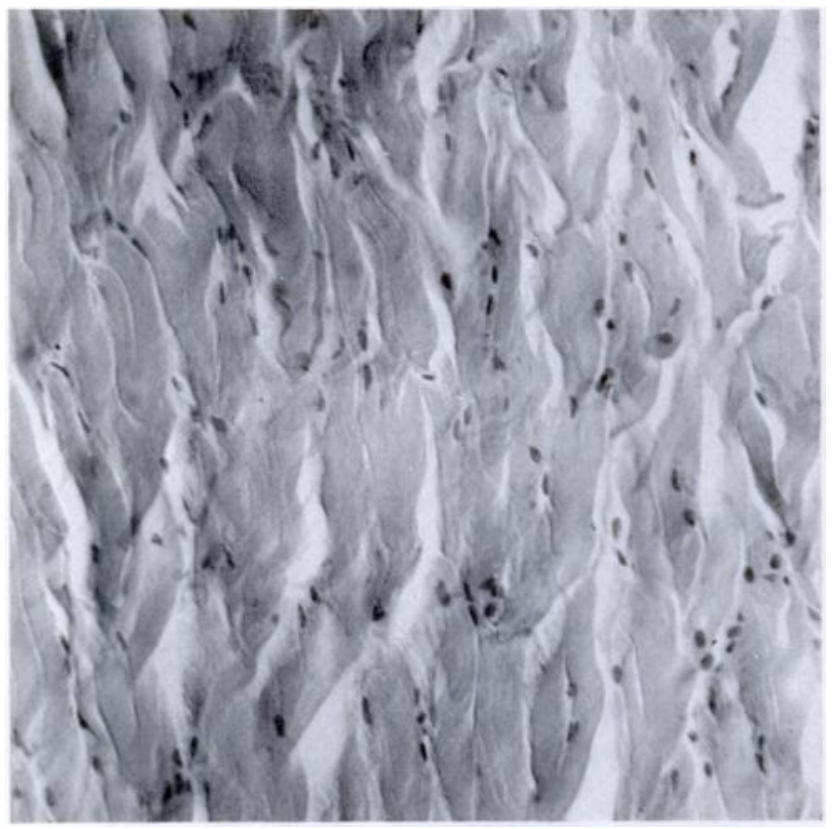

Fig. 1

Autogenous graft at two days. Section from the middle of the graft. The fibres remain intact. The nuclei are not pyknotic. There is slight oedema but no cellular exudate. (Haematoxylin and eosin, $\times 180$.)

One week-The graft displayed marked oedema and peripheral reaction but the adhesions were easily separated from it. There was a disorderly network of capillaries over the graft surface but no definite evidence of intratendinous healing. The joins were still well demarcated.

Microscopically, on the surface of the host tissue and graft, there was marked proliferation of capillaries and fibroblasts and moderate polymorphonuclear infiltration in this zone of reaction which was markedly thickened in comparison with the appearances at two days. The fibroblasts within this zone were arranged with their long axes in the long axis of the graft. Capillaries entered the periphery of the graft from the surrounding zone of reaction and were noted throughout the graft. There was similar capillary response in the host. A similar zone of organisation was present between ends of stumps and graft. There were increased numbers of nuclei within host and graft probably representing fibroblasts. In staining reaction the host tissue and graft varied from pale to deeply eosinophilic.

Two weeks-Macroscopically the graft showed continued increase in vascularity. The tendon ends were rounded and surrounded by scar whose fibres appeared oriented in the longitudinal axis of the tendon and the graft. The joins were still well demarcated.

Microscopically the zone of organisation appeared as broad as that at one week, but polymorphonuclear infiltration seemed less. There was no marked change in the amount of fibroblastic proliferation, but capillary proliferation in the graft appeared less. There was no change in capillary proliferation in the stumps. The cellularity of the graft seemed less than that at one week but that of the stumps seemed slightly more. The nuclei in the cells 
of the graft were not pyknotic. There was continued variability in staining of the collagenous fibres of graft and host. At the periphery of the zone of organisation there was an indefinite layer of loose areolar tissue which blended with the more compact zone of organisation.

Three weeks-The surfaces of graft and stumps appeared hyperaemic. The joins were indicated only by the peripheral silk sutures. The distal stump appeared moderately attenuated and was less vascular than the proximal stump. The surfaces of graft and host appeared moderately thickened but were shiny (Fig. 2).

Microscopically the zone of peripheral organisation appeared to be more compact. There was focal collagenisation in this zone. There were fewer capillaries in the zone, the line of demarcation between it and the graft was indistinct and there was blending of fibroblasts outside the graft with those proliferating within it. There was marked fibroblastic proliferation in the zone of organisation within the grafts and in the stumps and these fibroblasts were oriented longitudinally. Polymorphonuclear leucocyte infiltration was slight. Though the number of capillaries had decreased, the size of the individual vessels had increased. The orientation of vessels was more orderly and vessels traversing the zone of organisation between stump and host were seen. Some vessels possessed muscular coats. There was collagenisation within the graft and in the zone of organisation. There was blending of cytoplasmic staining reaction of the graft and the zone of organisation so that it was not possible to state where the graft terminated. There was an indefinite layer of loose areolar tissue peripheral to, and blending with, the dense zone of peripheral organisation.

Four weeks-There was loss of definition of the graft and further increase in vascularity, primarily in the region of the joins. The graft and tendon stumps appeared shiny but these surfaces appeared thicker than normal paratenon.

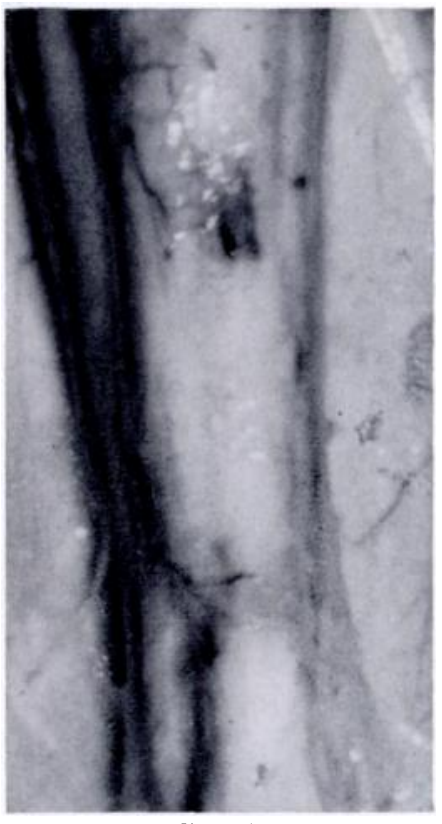

FiG. 2

Autogenous graft at three weeks. The graft is defined by the silk sutures and the joins are obliterated. There is diffuse mild hyperaemia over the surface of the graft with increase in vascularity near the joins. The surfaces of the graft are shiny and show minimal peripheral reaction.

Microscopically the graft remained hypercellular and comparable with that at three weeks. Both the zone of organisation and the stumps were relatively less cellular. In all areas there were fewer capillaries. They traversed the periphery of the graft and entered at either end. Some peripheral vessels had muscular coats. There was increase in collagenisation of the zone of peripheral organisation and graft. The stumps had the histological appearance of mature tendon. There was a peripheral areolar zone which blended indefinitely with the more compact zone of peripheral organisation. Collagenous fibres and fibroblastic nuclei between graft and host were mostly oriented longitudinally. The staining reaction was comparable with that at three weeks.

Five weeks-The scar over the tendon appeared shiny and thin. The adhesions about the tendon and its graft were thin and separated easily. The tendon stumps appeared well integrated with the graft and where

Autogenous graft at five weeks. The pattern of compact, paralle collagenous bundles is that of mature tendon. Widely separated capillaries persist. (Haematoxylin and eosin, $\times 180$.)

VOL. 45 B, NO. I. FEBRU ARY 1963 
separation had occurred the stumps had changed to the configuration of the graft. There was continued hyperaemia but the vessels appeared to be oriented longitudinally in the axis of graft and host.

Microscopically there was collagenisation of the zone of organisation between stumps and graft and of the stumps so that there was a uniform histological pattern

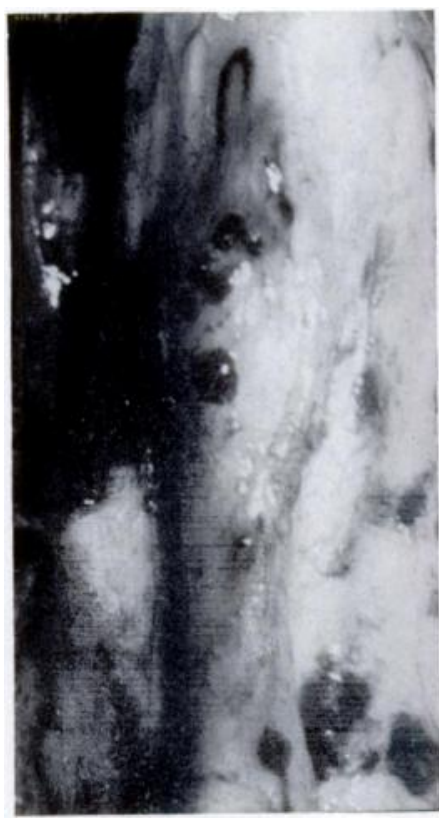

FIG. 4 throughout stumps and graft. The collagenous tissue occurred in bundles. Cellularity was uniform throughout the graft and stumps. There were few capillaries, and these did not show evidence of active proliferation. Small arterioles were noted peripherally (Fig. 3). Six weeks-The tendon was well defined and adhesions appeared less dense; the paratenon appeared shiny and thin. There was no specific pattern of vascularisation and there was only mild diffuse hyperaemia over surfaces of graft and host. The integration of tendon and graft was complete; where graft had pulled from host there was complete restoration of continuity of tendon and graft (Fig. 4).

Microscopically the graft appeared as relatively mature tendon characterised by transformation of the zones of organisation into parallel fibres of collagenous connective tissue which were oriented longitudinally and were indistinguishable from mature tendon except at the sites of sutures. Capillaries were fewer than at five weeks. They were distributed in orderly fashion between bundles of longitudinal fibres. The zone of loose areolar tissue was thin and sharply defined from the underlying tendon.

FIG. 4

Autogenous graft at six weeks. Integration of tendon and graft is complete. Diffuse mild hyperaemia persists. Surfaces of graft and tendon are shiny and show minimal reaction.

\section{HOMOGENOUS LYOPHILISED PRESERVED GRAFTS}

One week-The graft was well defined with no obvious healing at the joins. The tendon ends were rounded. There was minimal scarring at the periphery of the graft. There was mild capillary proliferation over the periphery of the stumps; there was none over the graft.

Microscopically the graft had only faintly staining "ghost" nuclei. It was sharply demarcated from the host tissue. The stumps were composed of compact collagenous connective tissue and cellularity did not appear increased. There was no peripheral zone of organisation. Between the stumps and graft there was moderate exudate of polymorphonuclear leucocytes and fibrin, with moderate proliferation of fibroblasts and capillaries. There were no capillaries in the graft nor any increased capillary proliferation within the stumps. The graft fibres were uniformly eosinophilic. The stump fibres were eosinophilic in varying degrees. Two weeks-There was marked scarring with obliteration of joins and marked disorderly increase in capillary proliferation. The graft was completely obscured by granulation tissue (Fig. 5).

Microscopically the cells of the graft showed no nuclei except superficially where there was focal fibroblastic and capillary proliferation from the zones of organisation around the graft and

FIG. 5

Lyophilised graft at two weeks. There is marked disorderly hyperaemia and reaction about graft and tendon and the graft is obscured.

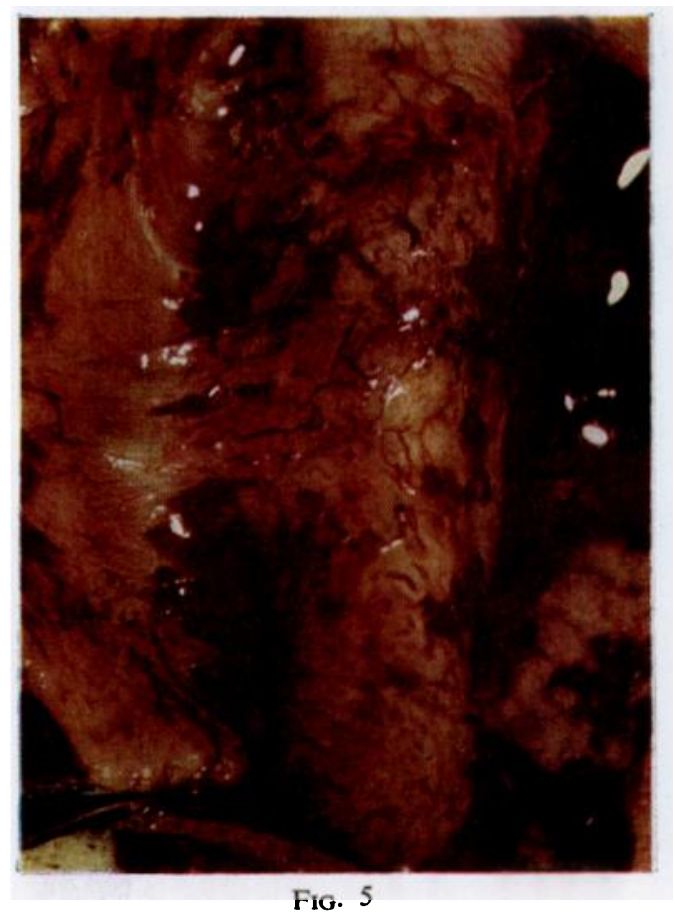

THE JOURNAL OF BONE AND JOINT SURGERY 
between graft and stumps. There was a broad zone of proliferation of capillaries surrounding the graft and the stumps and lying between graft and stumps. The superficial layer of the zone of organisation was loose and areolar and blended gradually with the more compact zone. Polymorphonuclear infiltration in the zone of organisation was of the same degree as at one week. The stumps were hypercellular, the cells being predominantly fibroblastic and oriented longitudinally.

Three weeks-This rabbit died forty-eight hours before examination of the tendon. Where the graft and stump were separated, continuity was established by scar. The graft was well defined, there was no significant peripheral scarring, and the surfaces appeared shiny. Though the major artery and vein were engorged with blood there was minimal evidence of capillary proliferation over the stumps or graft.

Microscopically the cells of the graft remained for the most part without nuclei. Stumps were hypercellular but to a lesser degree than at two weeks. There was a broad zone of organisation between graft and stumps but the zone of organisation about the graft was less broad than that noted at two

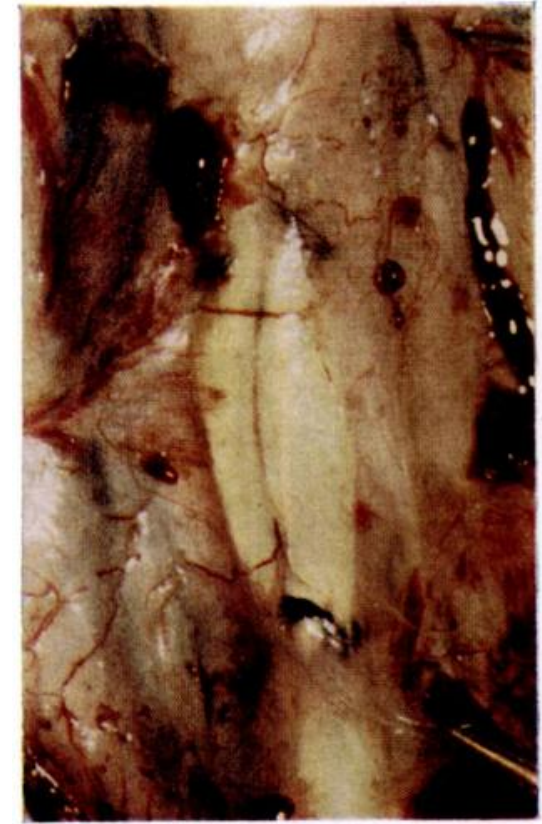

FIG. 6

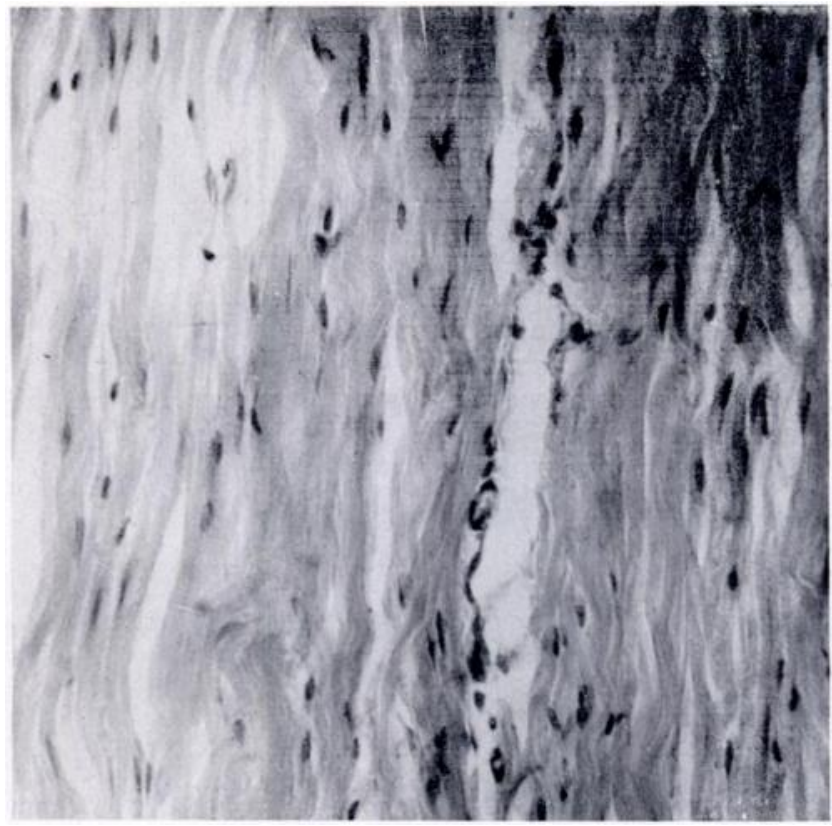

Fig. 7

Figure 6-Lyophilised graft at five weeks. The graft remains well defined but with healing across the joins. There is moderate peripheral reaction but only mild hyperaemia. Figure 7-Lyophilised graft at five weeks. The collagenous bundles are moderately compact and are interrupted by occasional capillaries. (Figure $7-$ Haematoxylin and eosin, $\times 180$.)

weeks. There was no increase in cellular proliferation or capillary proliferation within the graft. Exudation of polymorphonuclears was diminished. There was a thin areolar zone at the extreme periphery. Capillary proliferation in the stumps was essentially unchanged from that seen at two weeks. Four weeks-There was marked peripheral scarring with diffuse disorderly capillary proliferation. The joins were well healed and were defined only by the silk sutures. The periphery of the graft was not well defined because of the intense scarring.

Microscopically the graft and stumps appeared hypercellular. There were broad zones of organisation composed of proliferating fibroblasts and capillaries between the graft and stumps, and around the graft and stumps. There was longitudinal orientation of the fibres and nuclei of the fibroblasts in the zone of organisation and in the graft. Exudation of polymorphonuclear leucocytes was minimal. There was a loose areolar peripheral zone which blended with the zone of circumferential organisation. Fibres within the graft and stumps appeared deeply eosinophilic; the fibres in the zone of organisation did not exhibit collagenisation.

Five weeks-The graft was well defined. The joins were well healed. There was still fairly marked scarring but the surfaces appeared shiny. The overgrowth of scar was greatest in the region of the joins (Fig. 6).

VOL. 45 B, NO. 1, fEBRUARY 1963 
Microscopically the graft showed diminished cellularity; the fibres were collagenised and oriented in compact longitudinal bundles. There was slight collagenisation of the fibroblasts in the zone of organisation between graft and stumps and about the circumference of the graft. There was diminished cellularity in the zone of organisation. There was diminution in the number of capillaries in all areas and there were arterioles in the peripheral zone of organisation. There was minimal exudation of polymorphonuclear leucocytes in the zone of organisation and graft. There was no apparent change in the peripheral loose areolar tissue (Fig. 7).

Six weeks - Both joins were healed. There was no specific vascular pattern over the surfaces of graft or stumps. The peripheral scar was thin.

Microscopically there was focal collagenisation of the longitudinally oriented fibres in the zone of organisation. The dense collagenous connective tissue fibres of the graft were longitudinally oriented. Cellularity and capillary proliferation was diminished in the stumps, zone of organisation and graft. Cellularity was uniform and demarcation between zone of organisation, graft and stumps was no longer distinct. Leucocytes were very occasionally present in all areas. There was a thin, loose peripheral zone of areolar tissue which was sharply defined from the underlying circumferential zone of organisation.

Seven weeks-The graft was well healed and the joins were defined only by the silk sutures. There was mild hyperaemia over the stumps only. There was minimal scarring at the periphery of the graft and the surfaces appeared shiny.

Microscopically the presence of areas where the cells were without nuclei indicated incomplete organisation of the graft. In these zones the cells were basophilic and the fibres were fragmented. Alternating with them were longitudinal bundles of collagenous connective tissue which were hypercellular, especially those near the zones of necrosis. There were broad zones of organisation between stumps and graft. The pattern of collagenisation and cellularity of the circumferential zone of organisation was not uniform and adjacent to the foci of necrosis there was persistent proliferation of capillaries and fibroblasts. Adjacent to the regions of graft in which the cells had nuclei there was collagenisation of many of the fibres, with decrease in number of capillaries. Adjacent to the zones of necrosis there was focal infiltration of polymorphonuclear leucocytes. There was a thin, sharply defined areolar zone at the periphery overlying the stumps.

\section{HOMOGENOUS GRAFTS PRESERVED IN MERTHIOLATE}

One week-The graft appeared well demarcated from the surrounding tissue with no marked peripheral scarring. It appeared avascular and showed evidence of only mild superficial capillary proliferation. There was moderately dense scarring at the joins, but the line of anastomosis was still well demarcated (Fig. 8).

Microscopically, the cells of the graft showed no nuclei. Its periphery was infiltrated with polymorphonuclear leucocytes. There was an exudate of fibrin and leucocytes in the circumferential and intratendinous zones of organisation. By artefact the graft appeared to have become separated from these zones. In spite of this inflammation the graft was well apposed to the stumps. There was a broad zone of proliferating fibroblasts and capillaries infiltrating with polymorphonuclear leucocytes peripheral to the zone of inflammation. There was increased fibroblastic cellularity of the stumps with increased capillary proliferation. There was only focal infiltration of the stumps with leucocytes. There was a sharply defined zone of loose areolar tissue adjacent to the zone of organisation.

Two weeks-The graft showed marked peripheral disorderly vascularity with marked scarring. Its surface appeared dull. The joins were obliterated by scar and graft and tendon stumps appeared thickened. The surrounding superficial and deep fascia were thickened and oedematous.

Microscopically, the cells of the graft had nuclei only at the periphery where there was slight ingrowth of fibroblasts and capillaries from the circumferential zone of organisation and from the stumps. The circumferential zone of organisation was broad and hypercellular. Most of the proliferating fibroblasts were oriented in a longitudinal axis. There was hypercellularity of the stumps and capillary proliferation. There were foci of leucocytic infiltration and necrosis at the sutures, but exudation in other regions was diminished. There were arterioles in the circumferential zone of organisation, and peripheral to it there was a thin, ill defined zone of loose areolar tissue (Fig. 9).

Three weeks-Capillary proliferation persisted but was more orderly and was oriented in the long axis of the tendon. Peripheral scarring was less marked and the tendon surfaces were shiny. Continuity of graft and tendon was well established.

Microscopically the cells of the graft had nuclei only at the circumferential periphery and in the zones of organisation between stumps and graft. In the circumferential zone of organisation there was less capillary proliferation; there was longitudinal orientation of the fibroblasts whose nuclei were less closely packed. Arterioles were noted at the periphery of the zone of organisation. 


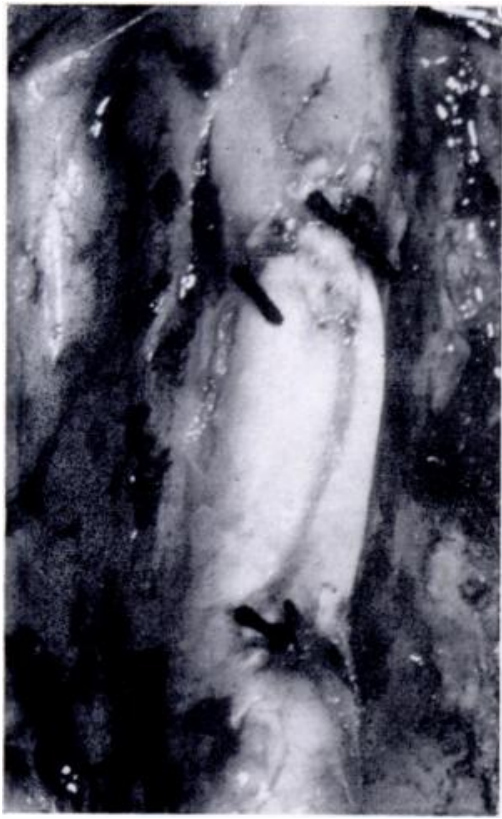

Fig. 8

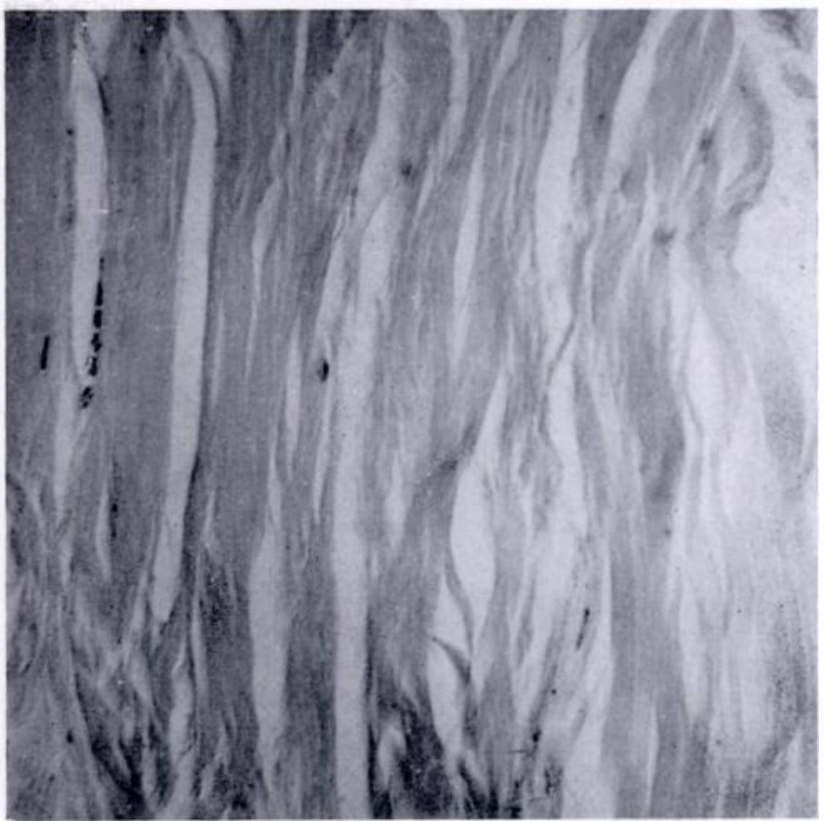

FiG. 9

Figure 8-Graft preserved in merthiolate at one week. The graft appears avascular and well demarcated. There is moderate peripheral reaction over the tendon stumps and at the joins. Figure 9-Graft preserved in merthiolate at two weeks. The collagen fibres from the donor have no nuclei. A few polymorphonuclear leucocytes are seen along one cleft between the fibres. (Figure 9-Haematoxylin and eosin, 180.)

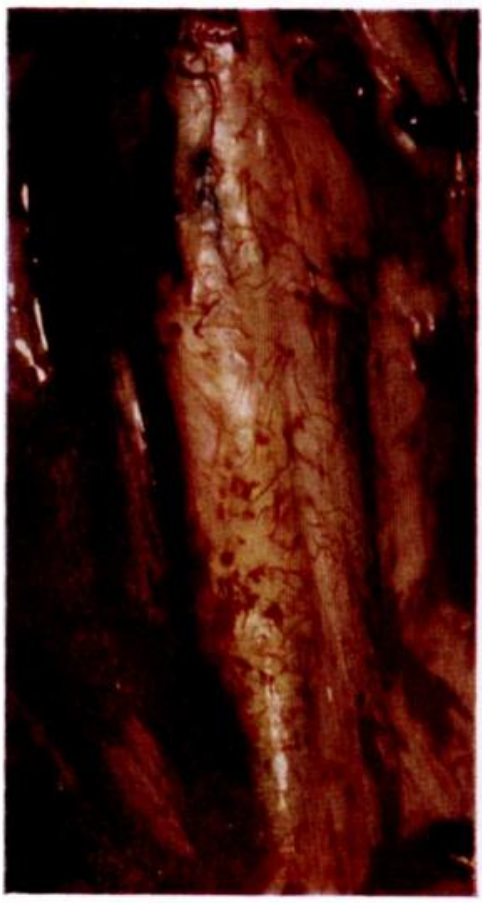

Fig. 10

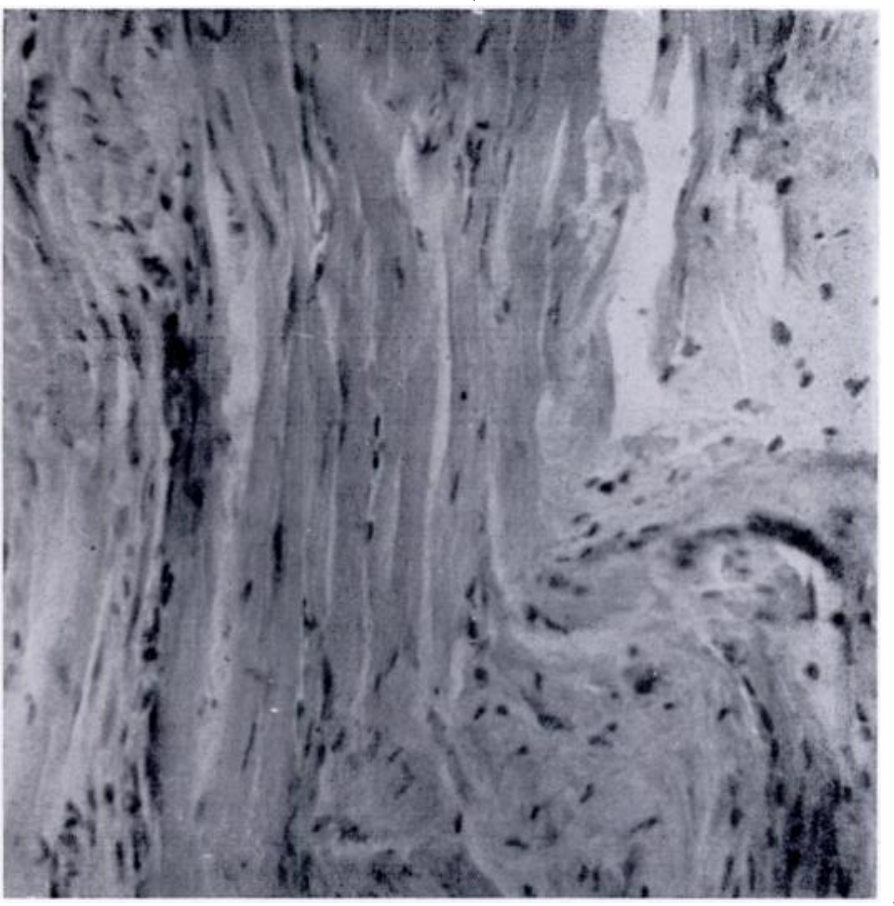

FIG. 11

Figure 10-Graft preserved in merthiolate at six weeks. There is marked peripheral disorderly hyperaemia and marked reaction. The graft is well healed at the joins and is defined only by the sutures. Figure $11-$ Graft preserved in merthiolate at six weeks. The bundles of compact collagenous connective tissue are oriented parallel to the long axis of the tendon. Capillaries and exudative cells are not prominent. (Figure 11Haematoxylin and eosin, $\times 180$.)

VOL. 45 B, NO. I, fEBruARY 1963 
Leucocytes were noted only adjacent to the sutures where there was focal necrosis. Stumps remained hypercellular but capillaries were less prominent than in the two-week graft. No zone of areolar tissue was noted at the periphery.

Four weeks-There was separation at the joins, with continuity of graft and stumps by dense scar. There was no specific vascular pattern over stumps or graft.

Microscopically the cells of the graft were without nuclei except in a few irregular foci where proliferating fibroblasts and capillaries had penetrated into the central portion of the graft both from the circumference and from the zones of organisation between stumps and graft. The circumferential zone of organisation was compact, fibres showed collagenisation and formation of bundles, capillaries were diminished in number and fibroblastic nuclei were oriented longitudinally. Polymorphonuclear leucocytes were rarely seen except at the sutures. The zone of loose areolar tissue was sharply defined and contained arterioles. Stumps were less cellular than noted in the three-week graft and capillaries were diminished in number.

Five weeks-There was excellent anastomosis of graft and stumps. There was continued hyperaemia over the graft and stumps and there was longitudinal orientation of the vessels. The surfaces were shiny.

Microscopically, there were nuclei throughout the graft continuous with the circumferential zone of organisation and the zone of organisation between stumps and graft. A few capillaries had penetrated the graft both from the circumference and from the zone of organisation between graft and stump. Both the zones of organisation showed collagenisation of fibres. Transition between the fibres of the stumps and of the graft and the zones of organisation was gradual. There was increased collagenisation of the fibres of the stumps with reduction of numbers of capillaries. Only rare leucocytes were noted in all areas. There was a zone of distinct loose areolar tissue along one margin of the graft.

Six weeks - There was marked hyperaemia of the surface of graft and host. Continuity of graft and host was well established. Scarring about the periphery of graft appeared less dense than at the joins (Fig. 10).

Microscopically, nuclei and capillaries were present in most of the graft but there were scattered foci without either nuclei or capillaries. In these regions fibres of the graft were deeply eosinophilic. Stumps and circumferential zones of organisation were uniformly collagenous, fibres in these regions were oriented longitudinally in compact bundles and only scattered capillaries were present. The graft remained well defined. Circumferential zone of organisation was compact and its fibres were collagenous and oriented in longitudinal bundles. There was a peripheral zone of loose areolar tissue which was well defined from the underlying compact area (Fig 11).

Seven weeks-The graft showed continued diffuse vascularity; the joins were well healed and were defined only by the peripheral sutures. Surfaces of graft and stumps were shiny.

The graft was cellular in most regions and scattered small capillaries were present. However, there were foci in which fibres of the graft had no nuclei and were brightly eosinophilic. Both ends of the graft were distorted by epithelial cysts lined by squamous epithelium and filled with keratin; this change was interpreted as a squamous metaplasia associated with reaction to suture material. There was collagenisation of both zones of organisation; the fibres were oriented longitudinally and arranged in bundles; there were only scattered capillaries in these zones and in the stumps. There were only rare scattered leucocytes except in the regions of the cysts. There was a sharply demarcated loose areolar zone peripherally.

\section{HOMOGENOUS ALCOHOL-PRESERVED GRAFTS}

One week-The graft was well defined and the joins had healed very slightly; they and the periphery of the graft were covered with granulation tissue. There was marked scarring over the periphery of the stumps with moderate erratic capillary proliferation (Fig. 12).

The fibres had no nuclei, were compact and shrunken. There was no proliferation of capillaries or fibroblasts into the graft. The zone of organisation immediately adjacent to the graft contained fibrin and cellular exudate. More peripheral to this region there was disorderly proliferation of fibroblasts and capillaries. There was marked hypercellularity of the stumps with infiltration of polymorphonuclear leucocytes and proliferation of capillaries and fibroblasts. Zone of organisation adjacent to the stumps exhibited disorderly proliferation of fibroblasts and capillaries with diffuse infiltration of polymorphonuclear leucocytes. There was a poorly defined zone of loose, hypercellular tissue adjacent to the more compact zone of organisation (Fig. 13).

Two weeks-The graft showed extremely marked peripheral scarring and was poorly defined. There was marked disorderly increase in vascularity over stumps and graft. The joins were obliterated by scar. 


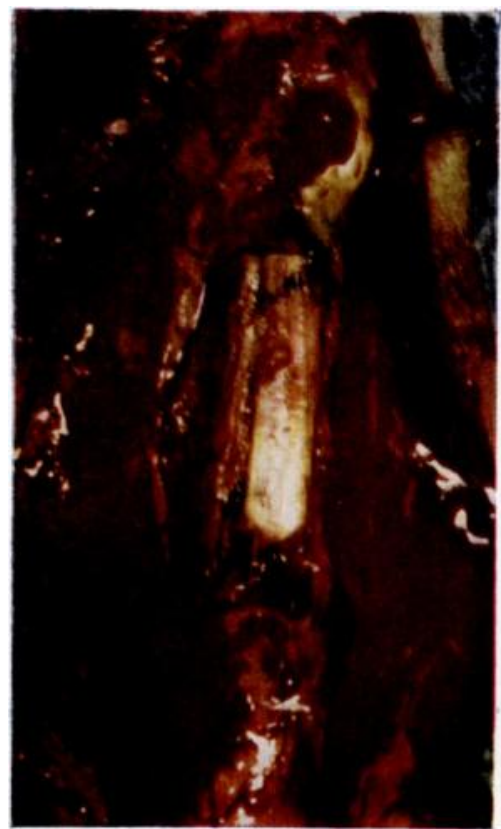

Fig. 12

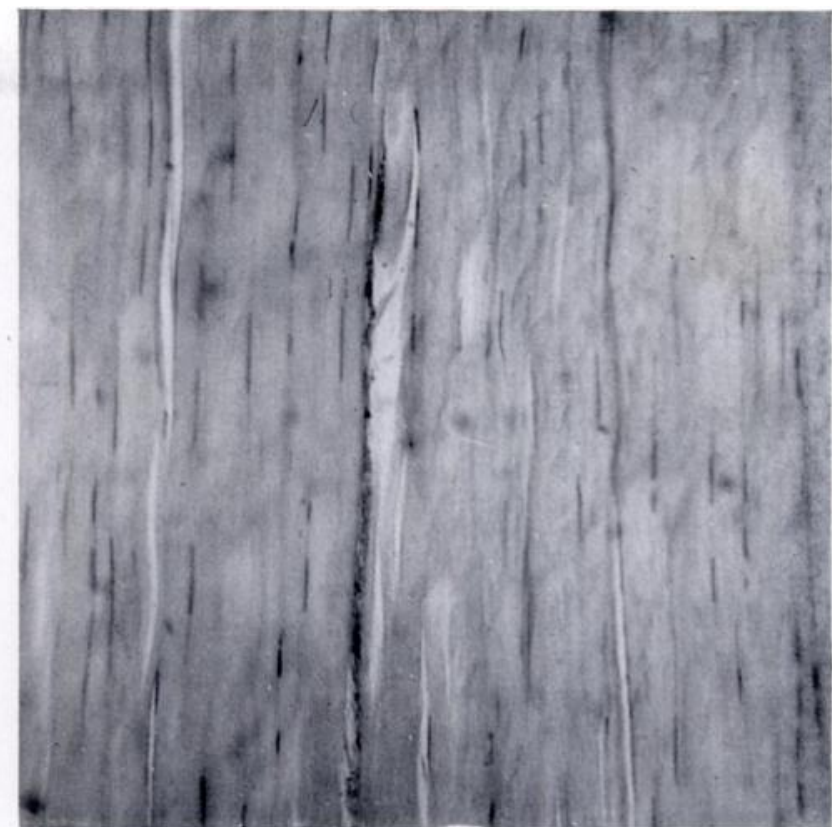

Fig. 13

Figure 12-Graft preserved in alcohol at one week. The graft is well'defined. At the joins the graft is covered with granulation tissue. There is marked peripheral hyperaemia over the stumps. Figure 13-Graft preserved in alcohol at one week. The compressed bundles of the denatured collagenous tissue of the graft are no longer eosinophilic. Pyknotic nuclei are occasionally seen. (Figure $13-$ Haematoxylin and eosin, $\times 180$.)

The cells of the graft had no nuclei. It was sharply demarcated from the zone of organisation except for fibroblastic proliferation immediately adjacent to the sutures. There was no evidence of fibroblastic proliferation into the graft peripherally or between graft and stumps. There was less leucocytic infiltration in the zones of organisation; in the circumferential zone of organisation fibres were oriented longitudinally and cellularity and number of capillaries were diminished. The stumps remained hypercellular and there was only slight polymorphonuclear leucocytic infiltration. Scattered capillaries were present in the stumps. There was a sharply defined loose areolar zone in which arterioles were present.

Three weeks-There was less peripheral scarring and the graft was well defined. There was slight increase in vascularity over its surface and marked increase over the stumps. There was granulation tissue about the sutures. The joins were well defined without marked proliferation of scar (Fig. 14).

Microscopically the cells of the graft had nuclei only at the circumference and at the ends where minimal focal proliferation of fibroblasts and capillaries had occurred from zones of organisation. There was focal collagenisation of the fibres in the zones of organisation and these were oriented longitudinally. There was less fibroblastic cellularity and capillary proliferation. There were rare polymorphonuclear leucocytes in all regions except in the graft. There was no zone of loose areolar tissue. Four weeks-The graft was moderately well defined. There was disorderly diffuse increase in vascularity with moderate peripheral scarring. There was apparent continuity of tendon and loss of definition at the joins.

FIG. 14

Graft preserved in alcohol at three weeks. The graft is well defined with minimal peripheral reaction. There is mild diffuse hyperaemia over its surface. The joins are obscured by granulation tissue.

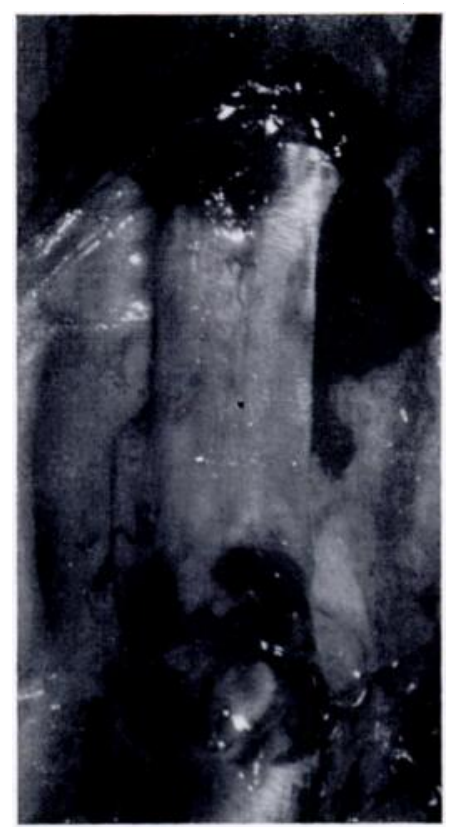

Fig. 14 
Microscopically the cells of the graft were mostly without nuclei. There were several broad regions of proliferation of capillaries and fibroblasts extending from adjacent zones of organisation into the graft. The fibres of the graft were fragmented and less compact. Zones of organisation were more compact with longitudinal orientation of the fibres and capillaries were scattered. There was focal collagenisation of fibres in the zones of organisation and the degree of fibroblastic proliferation was comparable with that seen at three weeks. Only scattered neutrophils were present. Transition between stumps and zones of organisation was indistinct. There was a distinct well defined zone of loose areolar tissue at the periphery.

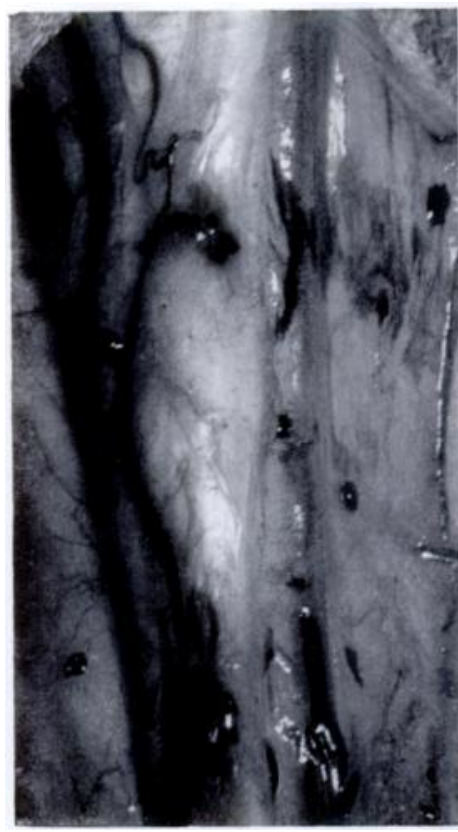

Fig. 15

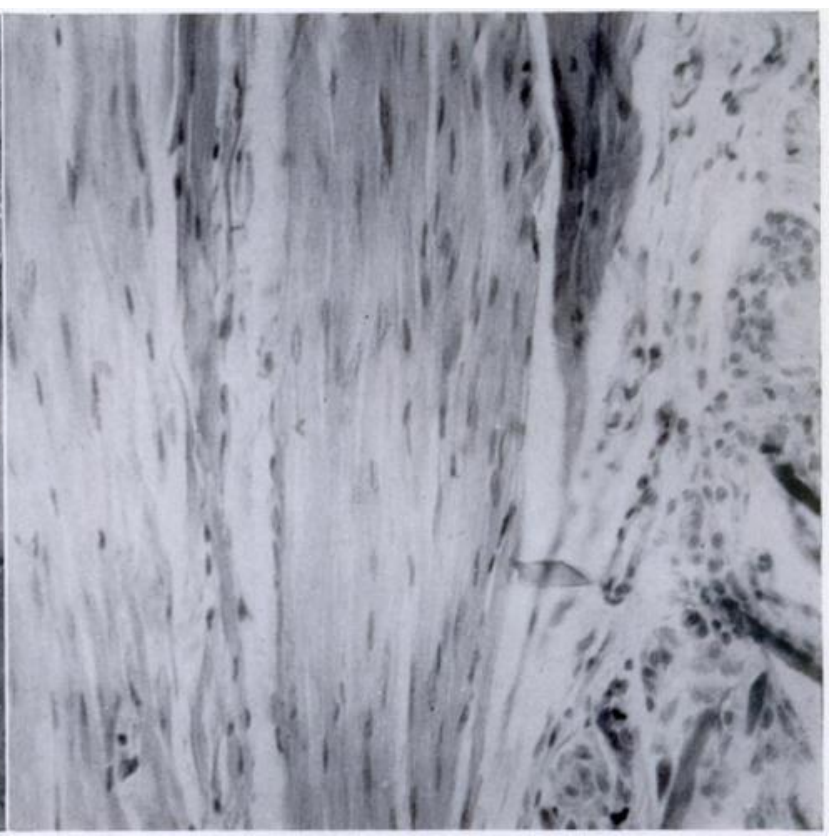

Fig. 16

Figure 15-Graft preserved in alcohol at six weeks. The graft and stumps appear well healed and the joins are defined mainly by the sutures. Surfaces of the graft and stumps are shiny and the peripheral scar appears loose. Figure 16-Graft preserved in alcohol at six weeks. The graft has the pattern of mature tendon. Foreign body reaction to fragments of suture is seen in the lower right part of the section. (Figure 16-Haematoxylin and eosin, $\times 180$.)

Five weeks-The graft still showed fairly marked peripheral scarring. There was continued peripheral vascular proliferation and the vessels were oriented longitudinally. The peripheral surfaces of graft and host were shiny. The joins were well healed and defined only by the silk sutures.

There were broad zones of organisation between stumps and graft. Otherwise, findings were essentially the same as those seen at four weeks.

Six weeks-The graft and tendon looked the same. Where there had been separation at the joins continuity had been preserved by axially oriented scar. There was no gross vascularity and the vessels were oriented axially. There was marked peripheral scarring and the surfaces appeared shiny (Fig. 15).

There was fibroblastic cellular proliferation throughout the graft. Most of the fibres in the graft had undergone collagenisation. Capillaries were scattered throughout it. Zones of organisation, stumps and ends of graft demonstrated continuity of the longitudinally oriented collagenous fibres. There were capillaries in the stumps. There was a thin, poorly defined zone of loose areolar tissue which contained arterioles. Leucocytes were rarely seen (Fig. 16).

Seven weeks-The graft displayed minimal peripheral scarring. Its surfaces were shiny and there was only slight hyperaemia. Vessels were longitudinally oriented. The joins were well healed.

Dense longitudinally oriented, collagenous fibres were present throughout the specimen and no graft fibres as such could be identified. Scattered capillaries were present throughout and there was a thin loose areolar zone. 


\section{DISCUSSION}

The vascular reaction-Mild vascular proliferation over the autogenous grafts and the host was seen as early as the second day. In the homogenous grafts comparable vascularity was seen only over the surfaces of the stumps and then only at the seventh day.

The surface of the autogenous graft at one week was obscured by an intensely vascular layer of granulation tissue which was loosely organised and without definite pattern. Comparable reaction in the lyophilised, merthiolate and alcohol grafts was not seen until the second week.

There was marked diminution in the surface vascularity of the autogenous grafts between the third and fourth weeks. In the lyophilised graft there was comparable diminution in surface vascularity only at the fifth week and in the alcohol graft only at the sixth week. The merthiolate graft continued to display moderate surface hyperaemia as late as the seventh week.

In none of the specimens was there evidence of production of a definite vascular pattern during the period of study. It appears that revascularisation follows a non-specific pattern and that proliferation of capillaries from the periphery of the graft and host extends into the region of the joins and into the circumferential areas of the graft and extends from tendon stumps across the joins into the graft. In the autogenous grafts vessels with muscular coats were seen traversing the zone of organisation between stumps and host as early as the third week. Not until the fifth to seventh weeks were similar vessels noted at the periphery of the homogenous grafts.

Fibroblastic reaction-In all specimens there was an intense peripheral fibroblastic reaction which developed alongside the vascular response. There was invariably some gap at the joins between proximal and distal stumps and the graft. These gaps were filled in by granulation tissue consisting of fibroblasts, capillaries and the fluid and cells of the exudate, all of which were derived from the host. There was a similar cuff of granulation tissue around the periphery of the graft. There was progressive organisation of granulation tissue and graft into compact, longitudinally oriented collagenous fibres and the graft and surrounding granulation tissue ultimately became invested in a thin layer of loose areolar tissue. This occurred in the autogenous grafts between the third and fourth weeks and in the alcohol and lyophilised grafts at the sixth week. The merthiolate grafts continued to exhibit moderate peripheral scarring as late as the seventh week.

The sequence of repair was similar in all types of preserved graft. There was growth of granulation tissue from the host between bundles of non-nucleated graft fibres. At first this ingrowth of fibroblasts and capillaries occurred slowly but from the third to the fifth week it went on rapidly. As the repair progressed, cellularity decreased and the new cells became oriented longitudinally into bundles of compact collagenous fibres. The process in the fresh grafts differed only in the initial stages where the interval between grafting and the period of hypercellularity was only one week.

Whether the collagen fibres in tendon grafts stay alive or die and are replaced cannot be determined from this study. The uniformity of staining reaction and maintenance of cellularity suggest that the fresh autogenous graft stays alive. However, this does not seem very significant, because ultimate healing only took three weeks longer in the lyophilised and alcohol-preserved grafts.

The cells of the graft-The cellular content of the graft varied according to the method of preservation. The cells of the fresh graft seemed to be intact even in the early stages and seemed to participate directly in the hypercellular response. As might be expected, the graft preserved in alcohol showed complete denaturation with shrinkage, loss of architecture and hyalinisation. The graft preserved in merthiolate lost all recognisable nuclei but did not show as much shrinkage or condensation as that fixed in alcohol. In each, the cells participating in the hypercellular response could only have been derived from the host. The lyophilised tissue 
retained nuclear " ghosts"--elongated, pyknotic cells, with beaded chromatin-which were recognisable until the new growth of tissue at the site of the graft appeared, but which could not be seen to participate in the formation of new cells. The rate of repair was slowed in those tissues which were treated with merthiolate or alcohol, or lyophilised. At from three to five weeks these grafts showed evidence of viability, as manifested by the presence of fibrocyte nuclei with distinguishable chromatin and recognisable cytoplasmic borders. The timing of these changes contrasts markedly with that of similar changes in the fresh grafts, in which repair began within the first week and had progressed to complete healing by three weeks.

The peripheral and junctural reactions were of course those seen in the organisation of any mesenchymal tissue after injury. The final stage of repair corresponds to the healed cicatrix of inflammation except that collagen bundles in these specimens exhibited the features of mature tendon, functionally, grossly, and histologically.

The development of tendinous structures possessing viable cells is interpreted as representing a specific type of maturation of multipotential fibroblasts in a zone of host reaction, rather than proliferation of a specific cell type, or tenoblast, from the parent structure of the host. The fact that alteration of environment will cause fibroblasts in tissue culture to assume different patterns favours this interpretation and makes it unnecessary to postulate a "blast" form for each specific tissue in the body (Maximow and Bloom 1952). As far as could be determined morphologically, cellular phagocytosis was not a major factor in the repair process in any of the grafts, nor was there evidence of enzymatic lysis of the fibres. Though the concept of creeping substitution provides a facile explanation for the eventual production of a structure indistinguishable from tendon, there is no definite evidence that such a process occurs.

\section{SUMMARY}

Comparison was made between the behaviour of fresh autogenous grafts of rabbit tendon and that of homogenous grafts inserted after the graft had been preserved for approximately one week, either in ethanol or merthiolate or by lyophilisation.

Regardless of the method of transplantation or preservation, a viable tendon-like structure of compact connective tissue bundles longitudinally oriented was eventually present at the sites of the grafts, with ingrowth of fibroblasts and capillaries from host to graft. The period between transplantation and recognisable viability of the grafts varied from less than one week for the autogenous transplants, to from three to five weeks for the preserved grafts.

Either autogenous or homogenous grafts will take in rabbits.

\section{REFERENCES}

Adams, W. (1860): On the Reparative Process in Human Tendons After Subcutaneous Division for the Cure of Deformities. London: John Churchill.

BeLtzow, A. (1883): Untersuchungen über Entwicklung und Regeneration der Sehnen. Archivfür Mikroskopische Anatomie, 22, 714.

Bizzozero, G. (1868): Sul processo di cicatrizzazione dei tendini tagliati. Annali universali di Medicina Milano, 203, 105.

Bloch, J. C., and Bonnet, P. (1929): Évolution et traitement des plaies des tendons de la main. Congrès Français de Chirurgie, $38 \mathrm{e}$ session, p. 547.

BORST, M. (1903): Ueber die Heilungsvorgänge nach Sehnenplastik. Beiträge zur pathologischen Anatomie und zur allgemeinen Pathologie, 34, 41.

Bunnell, S. (1956): Surgery of the Hand. Third edition, p. 457. Philadelphia: J. B. Lippincott Company.

Busse, O. (1892): Untersuchungen der feineren Vorgänge bei der Heilung von Sehnenwunden. Deutsche Zeitschrift für Chirurgie, 33, 30.

Creech, O., Jun., DeBakey, M. E., Cooley, D. A., and Self, M. M. (1954): Preparation and Use of Freezedried Arterial Homografts. Annals of Surgery, 140, 35.

Enderlen (1893): Ueber Sehnenregeneration. Archiv für Klinische Chirurgie, 46, 563.

THE JOURNAL OF BONE AND JOINT SURGERY 
Flynn, J. E., Wilson, J. T., Child, C. G., III, Graham, J. H. (1960): Heterogenous and Autogenous-Tendon Transplants. Journal of Bone and Joint Surgery, 42-A, 91.

Gallie, W. E., and LeMesurier, A. B. (1922): A Clinical and Experimental Study of the Free Transplantation of Fascia and Tendon. Journal of Bone and Joint Surgery, 4, 600.

Graham, W. C. (1955): Use of Frozen Stored Tendons for Grafting; An Experimental Study. Presented at Meeting of Orthopaedic Research Society, January 29, 1955. (As quoted by Bunnell, S. (1956).)

IMAYOSHI, M. (1925): Experimentelle Untersuchungen über Sehnenregeneration unter Anwendung der vitalen Carminspeicherungsmethode nach Kiyono. Archiv für Klinische Chirurgie, 137, 143.

KIRSCHNER, M. (1909): Ueber freie Sehnen und Fascientransplantation. Beiträge zur Klinischen Chirurgie, 65, 472.

LANGE, M. (1929): Die Naht und das Nahtmaterial in der Orthopädie. Zeitschrift für Orthopädische Chirurgie, Beilageheft, 51, 135. Stuttgart: Ferdinand Enke.

Marchand, F. (1901): Der Process der Wundheilung mit Einschluss der Transplantation. Stuttgart: Ferdinand Enke.

Mason, M. L., and Allen, H. S. (1941): The Rate of Healing of Tendons. Annals of Surgery, 113, 424.

Maximow, A. A., and Bloom, W. (1952): A Textbook of Histology. Sixth edition. Philadelphia and London: W. B. Saunders Company.

MAYER, L. (1921): The Free Transplantation of Tendons. American Journal of Surgery, 35, 271.

Migliavacca, A. (1925): Sull rigenerazione dei tendini. Bolletino della Società Medico-Chirurgica, Pavia, 38, 903. Neunof, H. (1923): The Transplantation of Tissues. New York: D. Appleton and Co.

Nisbet, N. W. (1960): Anatomy of the Calcaneal Tendon of the Rabbit. Journal of Bone and Joint Surgery, 42-B, 360.

PeAcock, E. E., Jun. (1959): Morphology of Homologous and Heterologous Tendon Grafts. Surgery Gynecology and Obstetrics, 109, 735.

Peacock, E. E. Jun., and Petty, J. (1960): Antigenicity of Tendon. Surgery, Gynecology and Obstetrics, $110,187$.

Peer, L. A. (1955): Transplantation of Tissues. Volume I. Baltimore: The Williams \& Wilkins Company.

ReHN, E. (1919): Zur den Fragen der Transplantation, Regeneration und Ortseinsetzenden functionellen Metaplasie. Archiv für Klinische Chirurgie, 112, 662.

SChradieck, C. (1900): Untersuchungen an Muskel Aund Sehne Nach Dur Tenotomie. Inaugural Dissertation to Faculty of Medicine. University of Rostock. 107 pages.

Schwarz, E. (1922): Úber die anatomischen Vorgänge bei der Sehnenregeneration und dem plastischen Ersatz von Sehnendefekten durch Sehne, Fascie und Bindgewebe. Eine experimentelle Studie. Deutsche Zeitschrift für Chirurgie, 173, 301.

SEGGel, R. (1903): Histologische Untersuchungen über die Heilung von Sehnenwunden und Sehnendefekten. Beiträge zur Klinischen Chirurgie, 37, 342.

Skoog, T., and Persson, B. H. (1954): An Experimental Study of the Early Healing of Tendons. Plastic and Reconstructive Surgery, 13, 384.

TenefF, S., and FondA, G. (1953): Innesti auto- omo- ed eteroplastici di tendine congelato. Minerva Ortopedia 4, 65 .

VACIRCA, M. (1954): Innesti di tendini eteroplastici refrigerati. Ricerche sperimentali. Archivio di Ortopedia 67, 458.

VIERING, W. (1891): Experimentelle Untersuchung über die Regeneration des Sehnengewebes. Archiv für pathologische Anatomie, 125, 252. 